\title{
OPTICAL AND RADIATIVE PROPERTIES OF INDIVIDUAL AND HYBRID NANOSUSPENSIONS: THE EFFECTS OF SIMILAR AND DISSIMILAR PARTICLE AGGLOMERATES ON THERMAL RADIATION
}

\author{
Layth Al-Gebory 1,2,*
}

\begin{abstract}
Nanosuspensions are proposed for use in improving the thermal efficiency of different thermal systems; including solar thermal power plants. Because of their excellent and unique thermo-optical properties, which are the basis of thermal transfer phenomena, they are used as working fluids in solar thermal collectors for photothermal energy conversion. However, particle agglomeration in nanosuspensions remains one of the most important challenges faced in terms of their usage. The purpose of this study is to investigate the particle agglomeration behavior of water-based $\mathrm{Al}_{2} \mathrm{O}_{3}$ and $\mathrm{TiO}_{2}$ individual and hybrid nanosuspensions and observe their effects on spectral radiative properties. By carrying out number of experiments, the effects of similar and dissimilar particle agglomerations on radiative properties are clarified. The results show that $\mathrm{pH}$ have significant effect on the particle agglomeration which in turn affects the optical and radiative properties involving individual and hybrid nanosuspensions. Particle agglomerates (similar and dissimilar) plays an important role when specific radiative properties are required for specific applications. Different regimes of the dependent/independent scattering can be obtained from the effect of similar and dissimilar particle agglomerates of a particular nanosuspension.
\end{abstract}

Keywords: Radiative properties; particle agglomeration; nanosuspensions; dependent-independent scattering regimes.

\section{INTRODUCTION}

Due to the increasing concerns regarding global climate change issues, the use of renewable energy sources for power generation is becoming a necessity. New and different renewable energy modalities mean that solar energy is the most attractive method in most regions of the world. In addition to the vast use of solar-PV (photovoltaic) systems, solar- thermal applications are also becoming widely available. In time, their prices will unavoidably drop and their reliability will increase. Unsustainable energy resources are unstable and expensive and creates this gives a new trend for the reliance of modern technologies and engineering applications of renewable energy resources particularly solar thermal collectors [1,2]. They are used for solar energy concentration, which is the most important part in solar thermal systems. Due to the low solar absorption of conventional working fluids, the efficiency of these collectors is usually low. Referring to the progress in nanotechnology over the past few years or so, applications of suspended nanoparticle in solar thermal collectors can be proposed. Nanoparticles are found to be an effective electromagnetic wave absorber within the UV-Visible wavelength range, where short wavelength radiations include high energy photons. It is worth mentioning that $85 \%$ of solar energy is dissolved within the UV-Visible range; while, conventional base fluids absorb about $15 \%$ of it within the infrared region $[3,6]$.

Nanosuspensions are defined as the suspension of nanoparticles with a size smaller than $100 \mathrm{~nm}$ in a base fluid. They play a significant role in different modern applications and processes; specifically, in the thermal field because of their unique thermophysical and radiative properties [7,8]. A homogeneous nanosuspension with one type of nanoparticle does not necessary include all the required characteristics for thermal, optical, and rheological applications, etc. Then, nanosuspensions can be prepared using different types of nanoparticles to tune the required properties for a specific application in hand. A homogeneous nanosuspensions with one type of nanoparticle does not necessary include all the required characteristics for thermal, optical, and rheological applications, etc. Hybrid

This paper was recommended for publication in revised form by Regional Editor Erdal Çetkin

${ }^{1}$ Department of Mechanical Engineering, Ozyegin University, Istanbul, TURKEY

${ }^{2}$ Department of Materials Engineering, University of Technology, Baghdad, IRAQ

*E-mail address: 130006@uotechnology.edu.iq, layth.ismael@ozu.edu.tr

Manuscript Received 31 May 2019, Accepted 28 November 2019 
nanosuspensions have combined chemical and physical properties of different types of nanoparticles. Therefore, hybrid nanosuspensions bring promising heat transfer applications, which are of significant importance to a variety of industrial sectors such as transportation, power generation, micro-manufacturing, electronics, engines, thermal therapy, heating, cooling, ventilation and air conditioning. The effectiveness of solar thermal systems used for photothermal energy conversion is measured by their ability of absorb radiative energy by the working medium; for such applications hybrid nanosuspensions are much better choice than traditional fluids [1]. However, the behavior of hybrid nanosuspensions in a working environment has not yet been studied extensively. Some hybrid nanosuspensions are expected to yield better thermophysical and radiative properties compared with individual nanosuspensions, and they offer a potential for thermal applications; including solar thermal systems or other applications that require desired and tunable radiative properties [9-13]. Dissimilar nanoparticles in the same medium behave differently from their individual existence. Therefore, it is important to investigate the stability behavior and its impacts on the radiative properties of hybrid nanosuspensions.

Nanoparticles in base fluids tend to agglomerate, and one of the most important challenges in the nanosuspensions research field is producing well dispersed and long-term stable (low particle agglomeration ratio) nanosuspensions. The stability of nanosuspensions is affected by different parameters such as the $\mathrm{pH}$ level of the suspension, particle size and shape, surfactant concentration, and sonication time [14-16].

A considerable number of investigations have been carried out on nanosuspensions over the last few decades, including their synthesis and characterization of properties for different applications [17-23]. Nanosuspensions are introduced for direct absorption solar collectors of many different types of nanoparticles which are experimentally investigated upon, such as carbon nanotubes, and metal oxide, etc. The effects of these nanoparticles on the collector's efficiency are studied [24]. The efficiency of the direct absorption solar collector is also examined, where water-based aluminium nanoparticles are used as a working fluid, and it has been reported that an improvement in the collector efficiency by up to $10 \%$ is proposed [25]. Nanosuspensions performance in solar collectors have been studied by Luo et al., and an improvement in the collector efficiency was observed up to approximately $2-25 \%$ as a result of the effect of adding nanoparticles [26]. $\mathrm{TiO}_{2}$ nanoparticles have attracted attention not only because of their radiative and thermophysical properties, but also because they are very suitable for many applications, and in a wide range of applications such as energy production (solar thermal systems) [27].

Radiative properties of nanosuspensions have been widely investigated in recent years, and the effects of dependent scattering were investigated in a system consisting of two spheres at an arbitrary distance with the index of refraction typical for soot particles [28]. Dependent and independent scattering regimes, which were explained for closely packed systems, include 10 spherical particles. A methodology was suggested to identify the characteristics of the particle agglomerates and the agglomeration process [29]. Dependent and independent scattering regimes of agglomerates have also been studied by a number of researchers [30-32].

In the current study, the investigation of water based $\mathrm{Al}_{2} \mathrm{O}_{3}$ and $\mathrm{TiO}_{2}$ individual and hybrid nanosuspensions is carried out for two objectives. Firstly, the characterization of the particle agglomeration to improve the stability of such nanosuspensions. Secondly, to explain the effects of the particle agglomeration on the radiative properties. In addition, the boundaries of the dependent and independent scattering regimes of such nanosuspensions are specified. An experimental investigation is completed to demonstrate the phenomena of similar and dissimilar particle agglomeration and as a result, the particle size distribution, which has a considerable impact on the radiative properties, and consequently on thermal radiation. The experiments are carried out by a number of sequential measurements in different conditions.

\section{EXPERIMENTS}

\section{Preparation of Nano Suspensions}

Two types of nanoparticles are used in this study; alumina $\left(\mathrm{Al}_{2} \mathrm{O}_{3}\right)$ with $99 \%$ trace metals basis with average diameter $40 \mathrm{~nm}$, and titania $\left(\mathrm{TiO}_{2}\right.$, anatase) with $99.5 \%$ trace metals basis with an average diameter of $40 \mathrm{~nm}$. These nanoparticles are obtained from the same source (Nanografi, Turkey). The two-step approach is used for the preparation of nanosuspensions. The nanoparticles are synthesized first by physical or chemical methods as dry 
powders, and then they are dispersed into the base fluids. This is the most economical and most popular method for preparation; however, it leads to a high rate of particle agglomeration.

The ultrasonic homogenizer is used to break large particle agglomerates that occur in the nanosuspensions. Ultrasonic energy is a form of mechanical vibratory energy, and its application over time leads to uniformly dispersed and stable nanosuspensions. To ensure that the nanoparticles are well dispersed into the suspensions, all the prepared samples were aged overnight in a magnetic stirrer at room temperature.

There are two important techniques to enhance the stability of nanosuspensions: steric and electrostatic stabilization. The electrostatic stabilization of nanosuspensions depends on many parameters such as $\mathrm{pH}$, temperature, zeta potential, and particle size, while the $\mathrm{pH}$ and zeta potential are the dominant parameters. It is usually preferred in thermal applications, specifically in the applications that include high temperatures, and the steric stabilization (the addition of a surfactant) leads to the contamination of the heat transfer medium (surfactants produce foams when heating). Therefore, in the solar thermal collectors where different temperature gradient occur, nanoparticle stabilization can be achieved by enhancing the repulsive forces between the particles, and this leads to a decrease in particle collisions; particularly at high temperatures. The comparison between the effects of the $\mathrm{pH}$ value and temperature shows that the $\mathrm{pH}$ carries the dominant impact on the particle agglomeration rate [18].

For this study, nanoparticles with volume fractions $(\varnothing)$ of $0.001 \%, 0.006 \%$, and $0.01 \%$ were monodispersed in distilled water (DI water) as a base fluid. Electrostatic stabilization was applied for the stability enhancement of the nanosuspensions, and the $\mathrm{pH}$ values of the suspensions were adjusted to 2,6 , and 10 , by using potassium hydroxide $(\mathrm{KOH})$, and hydrochloric acid $(\mathrm{HCl})$. The nanosuspensions zeta potential was measured as a function of the $\mathrm{pH}$, which provides a clear indication of the suspension's stability. Particle agglomeration can be investigated by using the dynamic light scattering (DLS) technique. This way, the average particle agglomerate size and particle size distribution of the nanosuspensions are obtained.

In the electrostatic stabilization, particle surfaces become charged in order to prevent their collisions (enhancing the repulsive force). Electrostatic stabilization of suspended particles is represented by zeta potential. For good stability, zeta potential value should be high $( \pm 30)$. Zeta potential is the electric potential in the double layer (ions layer) surrounding the suspended particles. If the suspension has a large absolute value of zeta potential, particles repel each other, while for a low absolute value of zeta potential the repulsive forces between suspended particles disappear and particle agglomeration is promoted. Within this unstable region, the particle agglomeration occurs at a higher rate and complex agglomerates are formed, thereby a significant increase in the particle size is observed.

\section{Measurements of Radiative Properties}

Radiative properties of particulate suspensions are sensitive to particle properties; including type, size, shape, concentration, and particle agglomeration, as well as the radiative properties of the medium. The spectral coefficients of the nanosuspensions are investigated in ultraviolet and visible wavelength ranges using ultraviolet/visible spectroscopy (UV-3600 Plus UV-VIS Spectrophotometer), and the calculations are based on the Beer-Lambert law. The scattering behavior is different for different particle agglomerates size and shape, where they play a significant role in the light attenuation and as a result, affect the spectral transmittance of the incident radiation $[12,13,16]$.

\section{RESULTS AND DISCUSSION \\ Characterization Of Nano Suspensions}

Nanoparticle suspensions with different particle agglomeration rates are considered. The goal is to explain the effect of the stability, average particle size, and particle size distribution on the radiative properties, and in addition, to explore the effect of the different particle agglomerate sizes on the dependent and independent scattering phenomena. All these parameters play a significant role in the photothermal applications and achieving long-term stable nanosuspensions remains one of the most important challenges.

For electrostatic stabilization, the particle surface charges to prevent particles collision and to decrease the agglomeration rate, where the dispersion stability enhance with the increasing $\mathrm{PH}$ value above, or a decrease of $\mathrm{PH}$ 
value below the isoelectric point (IEP). The IEP is the $\mathrm{pH}$ value (pHiso) at which a particle carries no net electrical charge (the point of zero charge), and the electrostatic stability vanishes. Colloidal suspensions become more stable against an agglomeration when the barriers (repulsive force) between the particles reinforce.

The photographs of the prepared nanosuspensions (individual and hybrid) inside clear glass containers are shown in Fig.1. The effects of different parameters (particle type, $\mathrm{pH}$, and $\emptyset$ ) on the stability behavior of the three types of nanosuspensions are explored, while the $\mathrm{pH}$ has significant effects in terms of the electrostatic stabilization of suspensions. A suspension with high stability means that the repulsive forces between the suspended particles are enhanced and become stronger than the attractive forces between them, and particles repel each other and stay in the suspension state for a long time. Thus, the $\mathrm{pH}$ value of the suspension should not be equal or close to the isoelectric point $\left(\mathrm{pH}_{\text {iso }}\right)$ of the nanoparticle, at which the particle carries no electrical charge to enhance the repulsive forces between the suspended particles. We observe from water based $\mathrm{TiO}_{2}$ nanosuspensions in Fig. 1(a) that at $\mathrm{pH}=6$ the suspensions have poor stability behavior, and a high level of particle agglomeration almost occurred, which leads to a high rate of particle sedimentation because the isoelectric point is $\mathrm{pH}_{\text {IEP }}=5-7$ for the $\mathrm{TiO}_{2}$ nanoparticle [33].

For water based $\mathrm{Al}_{2} \mathrm{O}_{3}$, which is shown in Fig. 1(b), the nanosuspensions also show poor stability behavior at $\mathrm{pH}=6$ and 10 , because the isoelectric point is $\mathrm{pH}_{\mathrm{IEP}}=7-8.5$ for the $\mathrm{Al}_{2} \mathrm{O}_{3}$ nanoparticle [34]. From Fig. 1(c), where it can be observed that the water-based hybrid $\left(\mathrm{TiO}_{2}+\mathrm{Al}_{2} \mathrm{O}_{3}\right)$ nanosuspensions display an enhancement in stability in comparison with the individual nanosuspensions in the same conditions; specifically, at $\mathrm{pH}=6$, which can be observed clearly in Fig. 2. Note that, different particles have their own chemical and physical properties and they display different behavior in the hybrid case than in their individual existence.

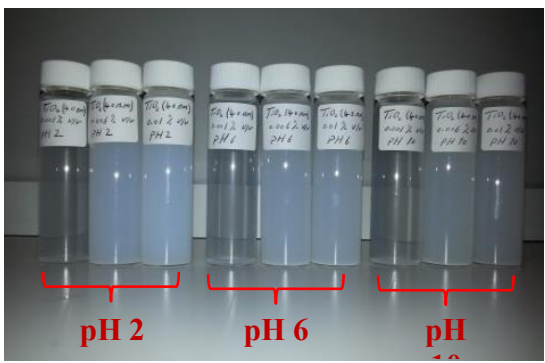

(a) Water based $\mathrm{TiO}_{2}$

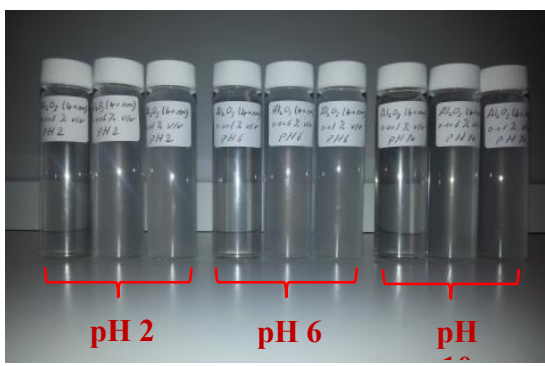

(b) Water based $\mathrm{Al}_{2} \mathrm{O}_{3}$

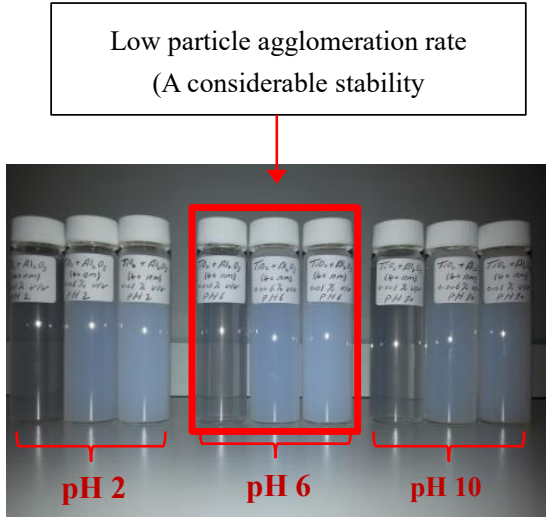

(c) Water based $\mathrm{TiO}_{2}+\mathrm{Al}_{2} \mathrm{O}_{3}$

Figure 1. Photographs of nanosuspensions at different conditions (Ø and $\mathrm{pH})$

In Fig. (2), the average particle agglomerate size (the diameter of agglomerate, $d_{a g g}$ ) is represented for the individual and hybrid nanosuspensions based on the DLS technique. In the DLS technique, the scattered radiation from all the particles and their agglomerates is correlated to the diffusion coefficient and based on the StokesEinstein equation for the spherical particle, and the radius of the particles and their agglomerates are estimated $[35,36]$. In the DLS technique, the average particle size (hydrodynamic diameter) is obtained from the particle size distribution (PSD), where small particles fluctuate the incident radiation more rapidly than the large particles. Particle agglomeration behavior can be investigated using the DLS technique, where the different sizes of particle agglomerates and the average (effective) particle agglomerate size can be obtained from the PSD of nanosuspensions. The results show that the individual and hybrid nanosuspensions have almost the same average particle agglomerates sizes at $\mathrm{pH}=2$. While, a considerable stability enhancement is obtained for the hybrid nanosuspensions at $\mathrm{pH}=6$, where the particle agglomerates have lower sizes than those of the individual nanosuspensions. In contrast, at $\mathrm{pH}=10$, the individual nanosuspensions have low particle agglomerates sizes, and then a better stability than those of the hybrid nanosuspensions can be observed. 


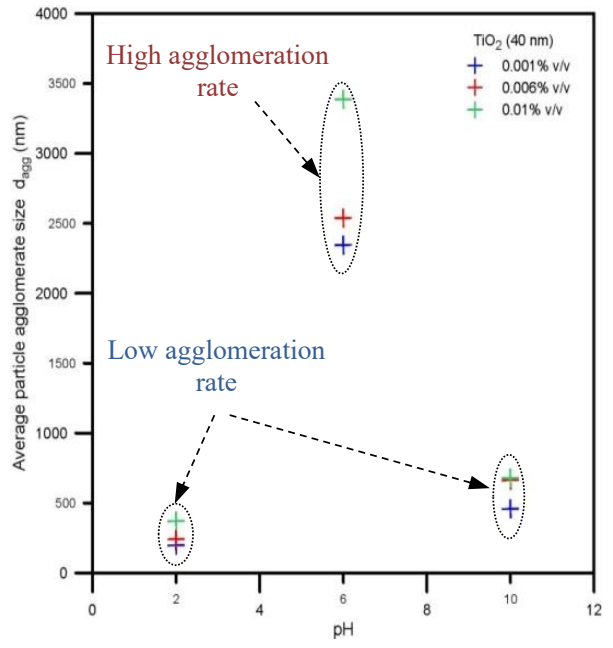

(a) Water based $\mathrm{TiO}_{2}$

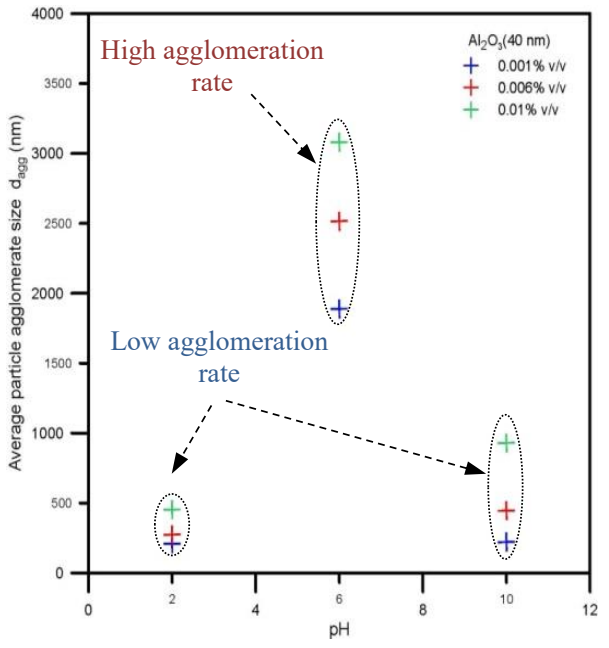

(b) Water based $\mathrm{Al}_{2} \mathrm{O}_{3}$

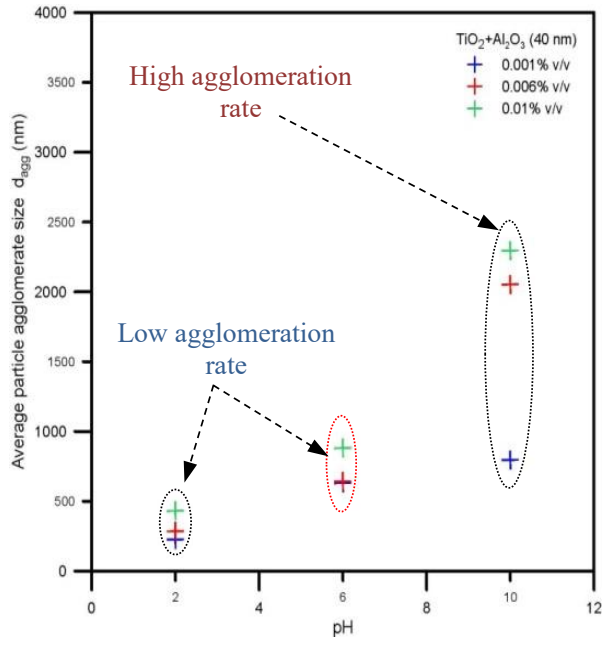

(c) Water based $\mathrm{TiO}_{2}+\mathrm{Al}_{2} \mathrm{O}_{3}$

Figure 2. Particle agglomeration curve of nanosuspensions

Fig. 3 shows the zeta potential curve of the nanosuspensions as a function of the $\mathrm{pH}$, where the zeta potential measurement provides a clear indication about the nanosuspensions stability. The zeta potential represents the potential difference between the charged particle and the medium layer that attached to the particle surface. In other words, the system the of electrical charge is modelled by the potential difference between the two considered spheres; the first one represents the charged particle and the second one represents the layer which includes the concentrated ions attached to the particle surface. According to the general chemistry principles of the particulate suspensions, the dispersion systems loses stability when the magnitude of the zeta potential has low values; either negative or positive (i.e. absolute value). In addition, there will be a region of zero zeta potential (i.e. IEP) for which the system is not particularly stable. Within this unstable region, a large rate of particle agglomeration can be achieved $[14,15]$.

In this figure, the zeta potential values of the hybrid nanosuspensions have positive values in all the $\mathrm{pH}$ values, which is different behavior to those of the individual nanosuspensions, where the nanoparticle surface charge and the surrounding electrostatic layer are different in different situations. Therefore, the hybrid nanosuspensions show a significant enhancement in the stability in comparison with the other two types at a specified $\mathrm{pH}$ value $(\mathrm{pH}=6)$. The results in Fig. 3 demonstrate the effect of the different parameters on the zeta potential, while the zeta potential value is affected primarily by the $\mathrm{pH}$.

The investigation of the particle size and size distribution (PSD) by using the light scattering technique has been studied by many researchers. For example, Aslan et al. used an Elliptical Polarized Light Scattering (EPLS) technique to investigate the structure and size of colloidal metal oxide [30].

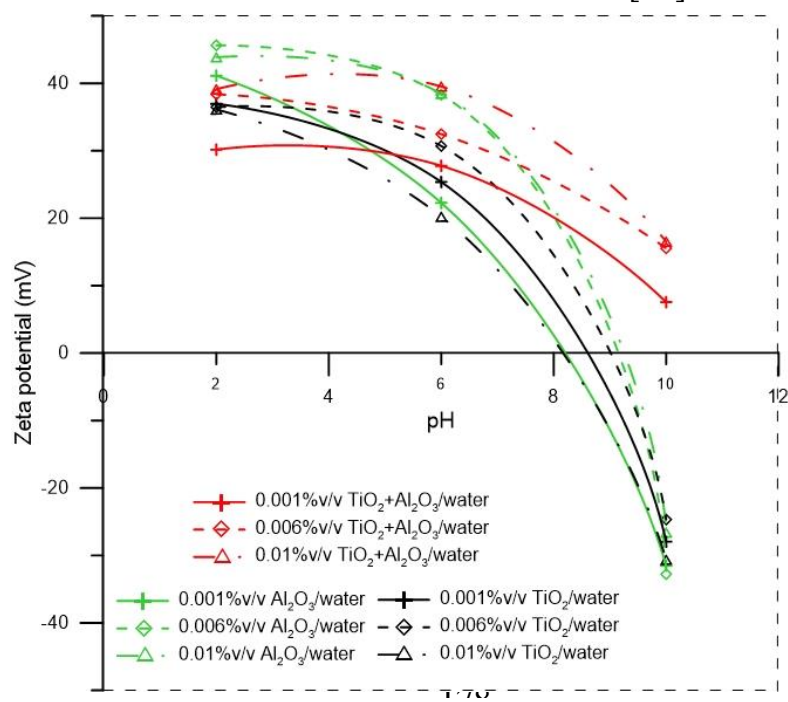

Figure 3. Zeta Potential curve of the nanosuspensions 
In this study, the particle size and size distribution (PSD) of nanosuspensions are investigated based on the DLS technique using Malvern Zetasizer nano ZS, and three readings were taken for each type of nanosuspensions to validate the results. In this paper, 27 types of nanosuspensions under different conditions were investigated, Fig. 4 shows three symbols of particle size distribution for three different types of nanosuspensions in the same conditions $(0.001 \% \mathrm{v} / \mathrm{v}$ and $\mathrm{pH}=6)$. The distribution of the particle size is displayed by a number of particles in these figures. The particle size increase from its original size is because of the particle agglomeration. The impact of the differing parameters on the particle size distribution can also be noted. Furthermore, the average (effective) particle agglomerate size under the effect of agglomeration for each kind of NPSs can be achieved by using this technique. All of the results are at a stable temperature equal to $25^{\circ} \mathrm{C}$.

The comparison between these three symbols shows that the hybrid nanosuspension Fig. 4(c) has a low average size of particle distribution, while the other two individual types Fig. 4(a) and 4(b) show a high average size of particle size distribution. Therefore, the hybrid nanosuspensions display a low average particle agglomeration rate in comparison with the other two individual types, which can be shown clearly in the average particle agglomerate size in Fig. (2).

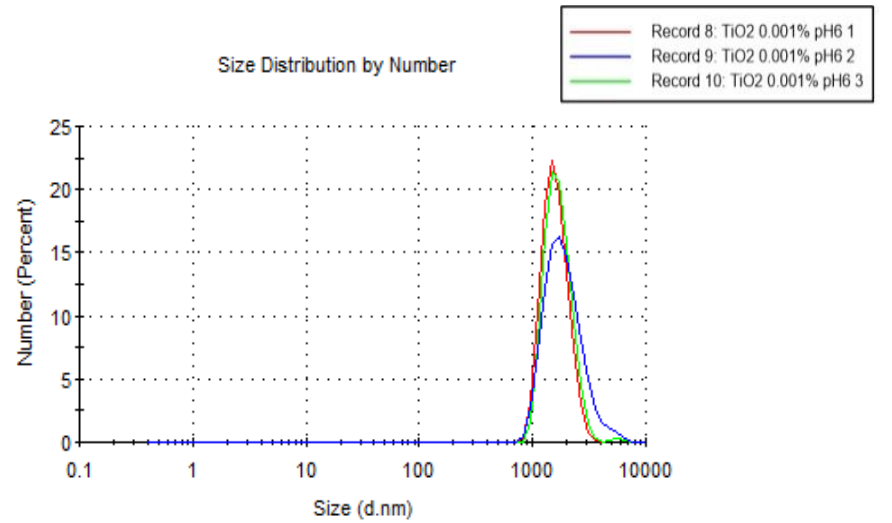

(a) Water based $\mathrm{TiO}_{2}$

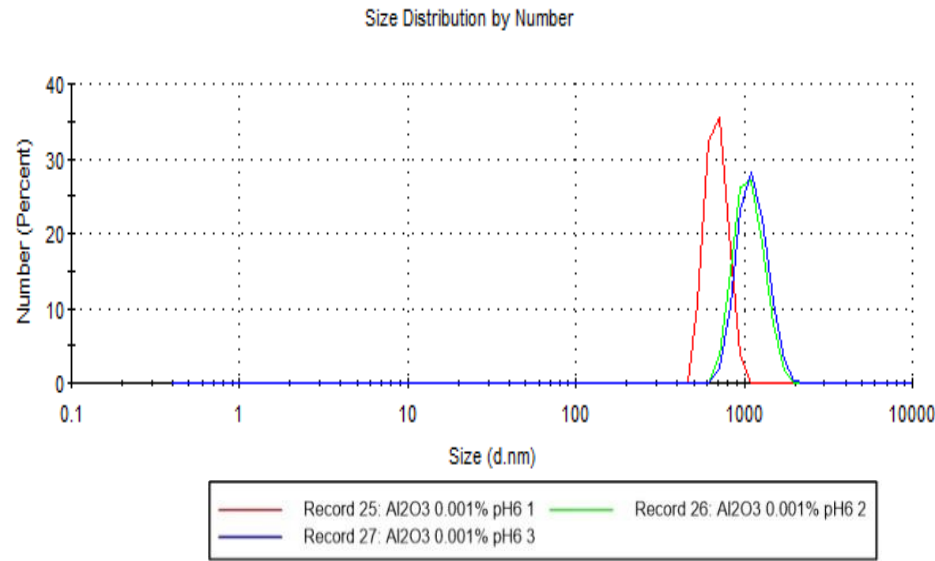

(b) Water based $\mathrm{Al}_{2} \mathrm{O}_{3}$

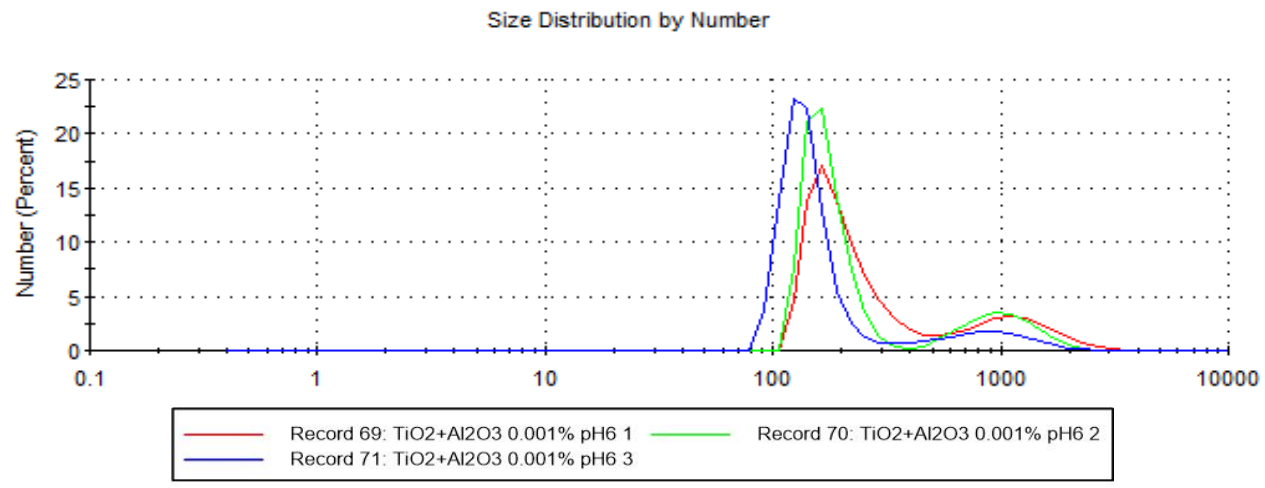

(c) Water based $\mathrm{TiO}_{2}+\mathrm{Al}_{2} \mathrm{O}_{3}$

Figure 4. Three symbols of particle size distribution for three different types of nanosuspensions at $0.001 \% \mathrm{v} / \mathrm{v}$ and $\mathrm{pH}=6$. The average particle size (a) $2354 \mathrm{~nm}$ (b) $1889 \mathrm{~nm}$ and (c) $631 \mathrm{~nm}$

\section{Radiative Properties}

The radiative properties of nanosuspensions are experimentally investigated in the UV/visible wavelength range. Since electromagnetic radiation has higher energy in this range, enhancing the radiative properties within this specification is desired for the photothermal energy conversion applications. Figs. 5 and 6 show the scattering and extinction (the particle scattering and medium absorption) coefficients of the nanosuspensions, which were obtained experimentally from the UV/Visible spectroscopy test at room temperature. The effects of the different parameters, 
which have been omitted in theoretical research, can be observed in this study. The $\mathrm{pH}$, particle agglomeration, particle size distribution, and concentration effects on the radiative properties are shown.

Figs. 5(a) and 6(a) represent the radiative properties of the water-based $\mathrm{TiO}_{2}$ nanosuspensions which are shown in Fig. 1(a). Figs. 5(b) and 6(b) represent the radiative properties of the water-based $\mathrm{Al}_{2} \mathrm{O}_{3}$ nanosuspensions which are shown in Fig. 1(b). Figs. 5(c) and 6(c) represent the radiative properties of the water-based $\mathrm{TiO}_{2}+\mathrm{Al}_{2} \mathrm{O}_{3}$ (hybrid) nanosuspensions which are shown in Fig. 1(c).

According to these results, the scattering and extinction coefficients reach their maximum at the ultraviolet radiation range (200-400 $\mathrm{nm}$ ) but they decrease in the visible range (400-800 nm). Generally, these coefficients are inversely proportional to the wavelength, and there is a considerable effect of particle volume fraction on all the types of nanosuspensions. The particle size has significant impacts on the radiative properties. The strength of the scattering is dependent on different factors which are the refractive indices of a particle and surrounding medium, particle size, and structure, as well as the wavelength of the incident radiation. When the particle size is similar to the wavelength, the scattering behavior is dominated by diffraction. For a small particle size, whose diameter is smaller than the wavelength, a strong scattering performance is obtained at a shorter wavelength. On the other hand, for the large particle $(x \gg 1)$ it removes twice the amount of radiation that is intercepted by the equivalent cross section area. Of these radiations passing within the equivalent cross section area, some will be refracted, and others reflected at the particle surface [13].

One of the most important observations is the effect of the dissimilar particles agglomeration on the scattering and extinction coefficient of the nanosuspensions, and these particles produce composite particle agglomerates including different optical properties to that of the existence of the individual particle. Thus, all the aspects of the particle agglomeration; including size and composition are considered in the investigation in addition to the other parameters. The oscillation in these figures for the water based $\mathrm{TiO}_{2}$ and $\mathrm{TiO}_{2}+\mathrm{Al}_{2} \mathrm{O}_{3}$ nanosuspensions in the ultraviolet wavelength range can be explained as the following. The oscillation produced from the interfaces of the refracted and transmitted radiation, and another effect is the multiple reflections from the surface of the particle agglomerates, the intensity of the reflected radiation increases with increasing the difference in the refractive indices of a particle and medium, and the intensity of reflected radiations increases with increasing the difference in the refractive indices of the particle and medium [16,37,38]. In addition, the high refractive index of a particle leads to increasing the oscillation, where the $\mathrm{TiO}_{2}$ particle has a refractive index three times that of the $\mathrm{Al}_{2} \mathrm{O}_{3}$ particle within this wavelength range. This affects the scattering efficiency, where different behavior can be observed from these two types of particles. It is observed that the oscillation decreases slightly in the hybrid nanosuspensions in comparison with the individual (water based $\mathrm{TiO}_{2}$ ) type. The scattered radiations display a complicated variation depending on the particle size and structure, the wavelength of the incident radiation and the complex refractive index of that particle at that wavelength. From Fig. 5, one can deduce how the scattered radiation must depend on the shape, and size, as well as its optical properties.

The absorption effect of the medium (water) is very low in comparison with the effect of the scattering effect in the extinction coefficient. Water is an effective media in the near infrared wavelength range, while, scattering is dominant in the ultraviolet-visible wavelength ranges. Therefore, the scattering and extinction figures look identical.

From Figs. 5 and 6, it can be observed that hybrid nanosuspensions show considerable radiative properties in comparison with the two individual types in addition to their better stability behavior at specified $\mathrm{pH}$ values, which allows them to be used in different applications. Light attenuation is one of the most important parameters for the solar thermal collectors. A particulate suspension with high light attenuation is desired for solar thermal utilization in terms of light-induced energy conversion. This is the principle of the direct absorption solar collectors, at which solar radiation incident and interacts directly with the working media. 


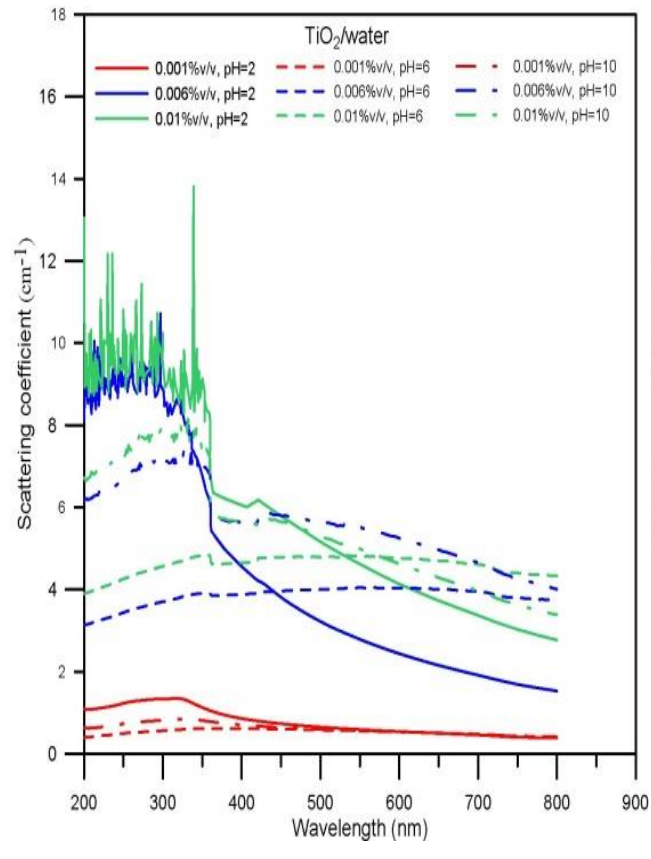

(a) Water based $\mathrm{TiO}_{2}$

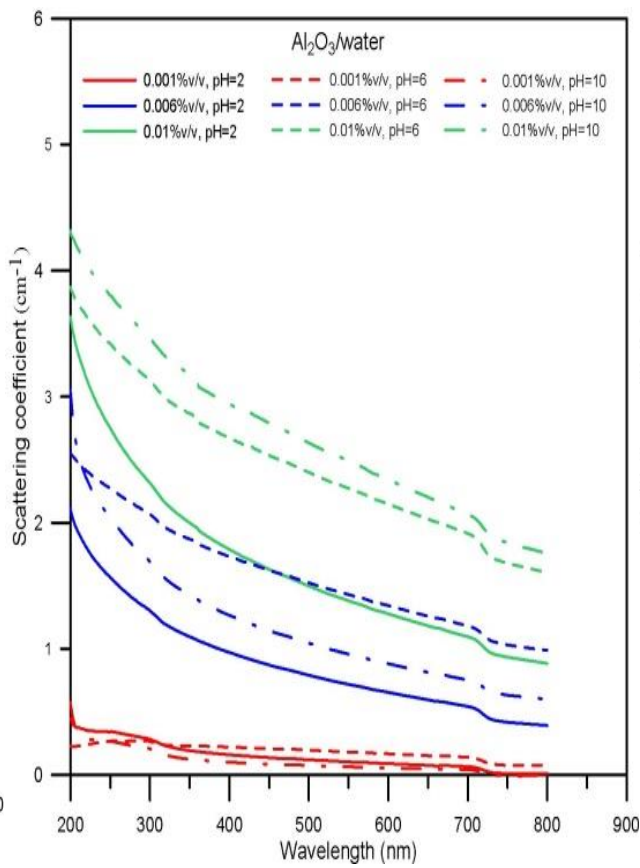

(b) Water based $\mathrm{Al}_{2} \mathrm{O}_{3}$

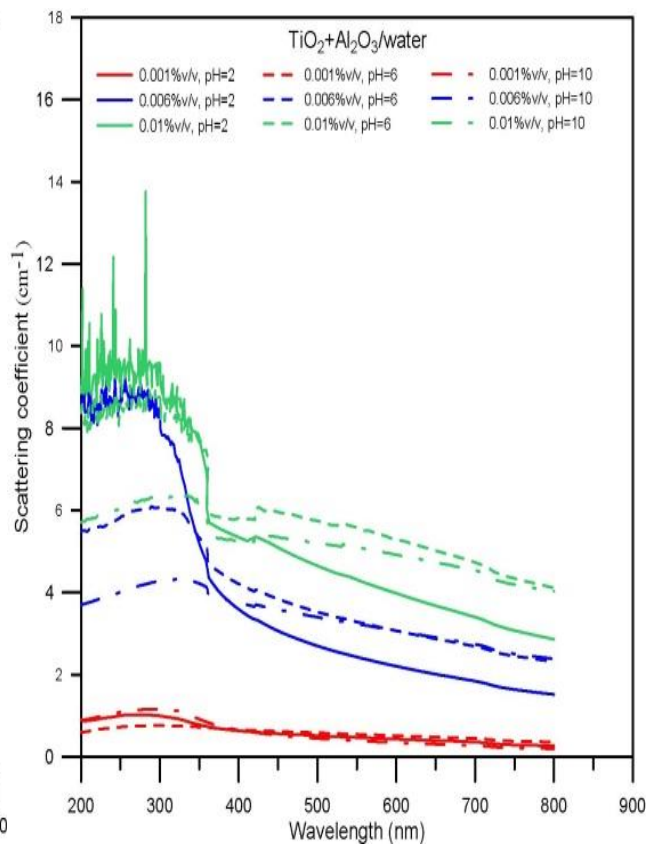

(c) Water based $\mathrm{TiO}_{2}+\mathrm{Al}_{2} \mathrm{O}_{3}$

Figure 5. Scattering coefficient of nanosuspensions based on UV-Visible spectroscopy test

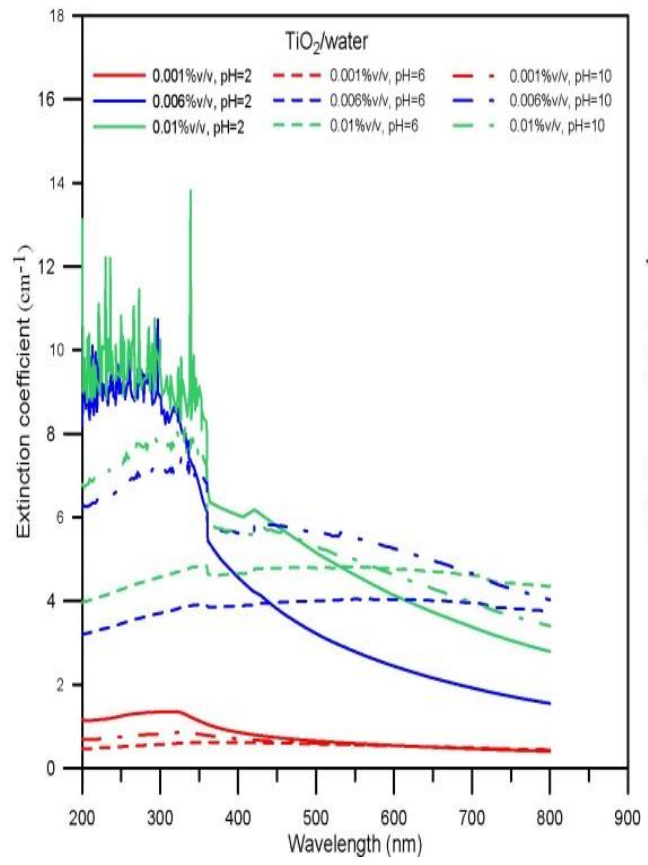

(a) Water based $\mathrm{TiO}_{2}$

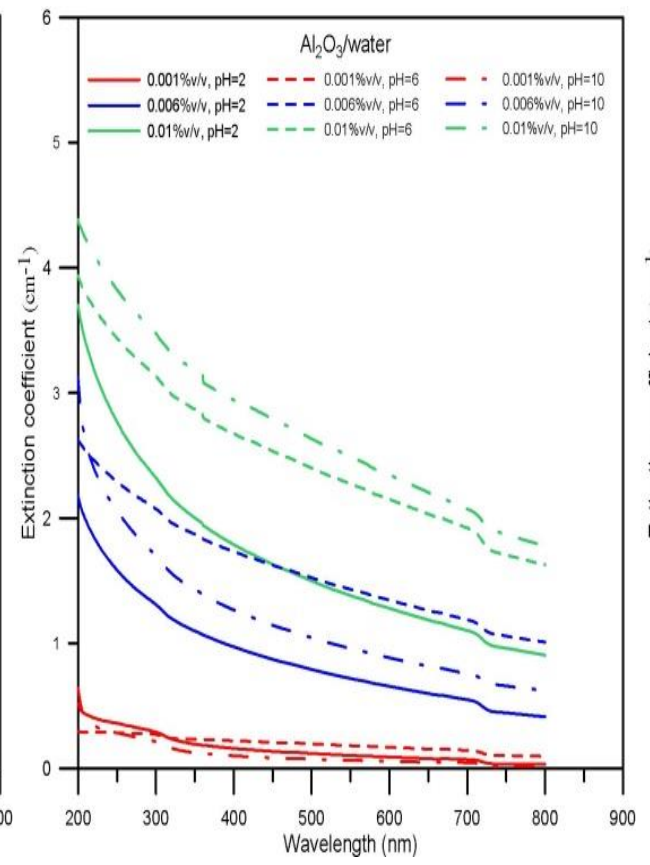

(b) Water based $\mathrm{Al}_{2} \mathrm{O}_{3}$

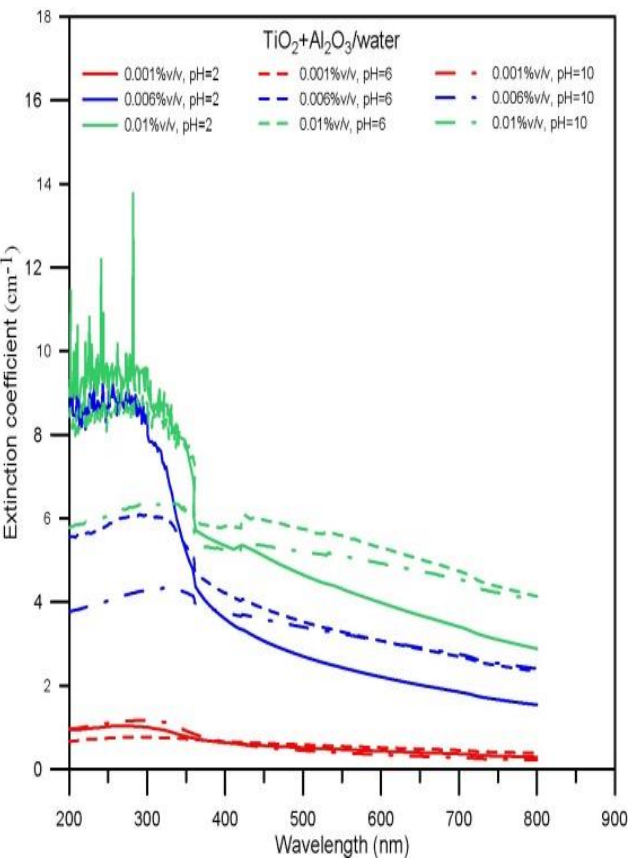

(c) Water based $\mathrm{TiO}_{2}+\mathrm{Al}_{2} \mathrm{O}_{3}$

Figure 6. Extinction (absorption and scattering) coefficient of nanosuspensions based on UV-Visible spectroscopy test 
Fig. 7 shows the boundaries of the dependent and independent scattering regimes for the water based $\mathrm{TiO}_{2}, \mathrm{Al}_{2} \mathrm{O}_{3}$ and $\mathrm{TiO}_{2}+\mathrm{Al}_{2} \mathrm{O}_{3}$ (individual and hybrid) nanosuspensions with nanoparticle size $\left(d_{p}=40 \mathrm{~nm}\right.$ ) and with a different particle volume fraction. Two regimes are specified for the nanosuspensions in this figure including with and without particle agglomeration regimes, and the calculations are specified at a UV-Visible wavelength range. The effects of particle size and size distribution, particle volume fraction, $\mathrm{pH}$ value, clearance the between the particles, wavelength, and particle agglomeration, are included in this figure where the experimental results are represented. $h / \lambda$ is the key parameter for the boundaries of dependent and independent scattering regimes. The experimental results display that the $h / \lambda$ is the most important parameter, where $h=f\left(\phi\right.$ and $\left.d_{p}\right)$ according to Woodcock's equation $[33,41]$.

In Fig. 7, the effect of the $\mathrm{pH}$ on the agglomeration behavior of nanosuspensions shows a different regime than that obtained from these nanosuspensions regardless of the particle agglomeration effect. The effect of the particle agglomeration in the same nanosuspensions conditions (particle type, $\phi$ and $d_{p}$ ) appears in the particle size distribution. Beyond any doubt, increasing the particle size results in increasing the clearance between the particle to the particle surfaces, which means that the effect of the dependent scattering disappears at the same wavelength. In the regime of the nanosuspension with the particle agglomeration, the independent scattering condition becomes $h / \lambda \gg 0.5$. In contrast, in the nanosuspensions without the particle agglomeration regime, the linear relationship between the particle size parameter and wavelength can be observed because the particle agglomeration is omitted, and the particles are assumed to have a fixed size $40 \mathrm{~nm}$.

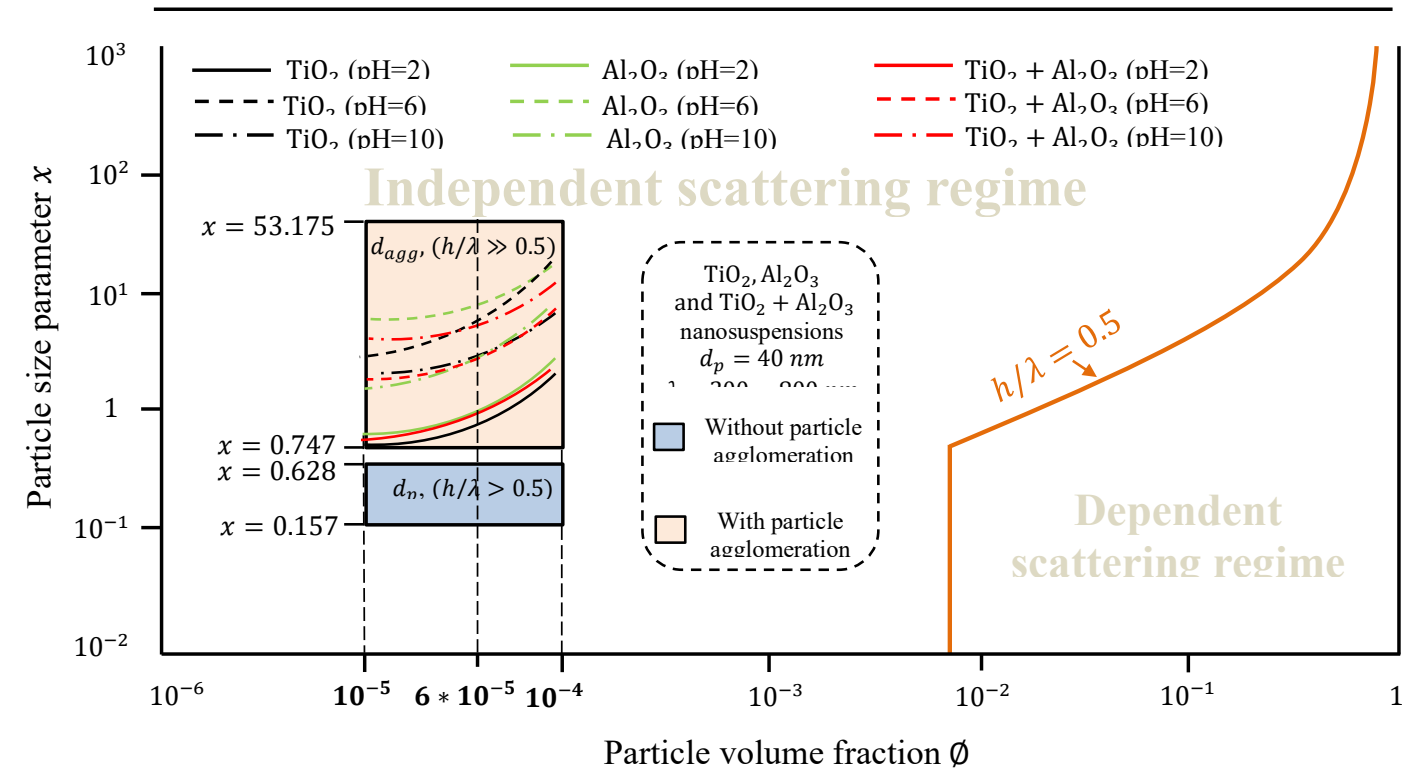

Figure 7. Independent and dependent scattering regimes including individual and hybrid nanosuspensions

Fig. 8 shows the boundaries of the independent and dependent scattering regimes for the individual and hybrid nanosuspensions. The relationship between the surface to surface particle distance and wavelength is represented in order to demonstrate the effects of the different parameters on the independent and dependent scattering regimes. As mentioned in the discussion of Fig. 7, the $\mathrm{pH}$ and $\varnothing$ parameters affect the particle agglomeration of the different nanosuspensions types (individual and hybrid), and particle agglomerates with different sizes are produced. These parameters with the optical properties of the nanoparticles affect the scattering phenomenon and its nature (dependent or independent) at specified wavelength ranges (UV/Vis wavelength ranges). Nanosuspensions approach the boundary between the independent and dependent scattering regimes with the increasing wavelength. Whereas the scattering behavior occurs in the independent scattering regime for all the types of nanosuspensions; this comes from the effects of the low particle volume fraction and particle agglomeration, which affect the clearance between the particles and consequently leads to $h / \lambda \gg 0.5$.

Fig. 9 shows the extinction coefficients of the nanosuspensions at the specified values of the $\mathrm{pH}$, and all the results are obtained at the wavelength value $\lambda=400 \mathrm{~nm}$. The results explain how the particle agglomeration affects the extinction coefficient of both the hybrid and individual nanosuspensions at the same wavelength. In fact, 
nanosuspensions show a different particle size distribution under a different condition where the particle agglomerate size is different. In addition, an extra influence from the composite particle agglomerates appears on the radiative properties of the hybrid nanosuspensions where each type of nanoparticle shows different optical properties. Therefore, different radiative properties (extinction coefficient) can be observed, which can be utilized in specific applications where desired radiative properties are needed.
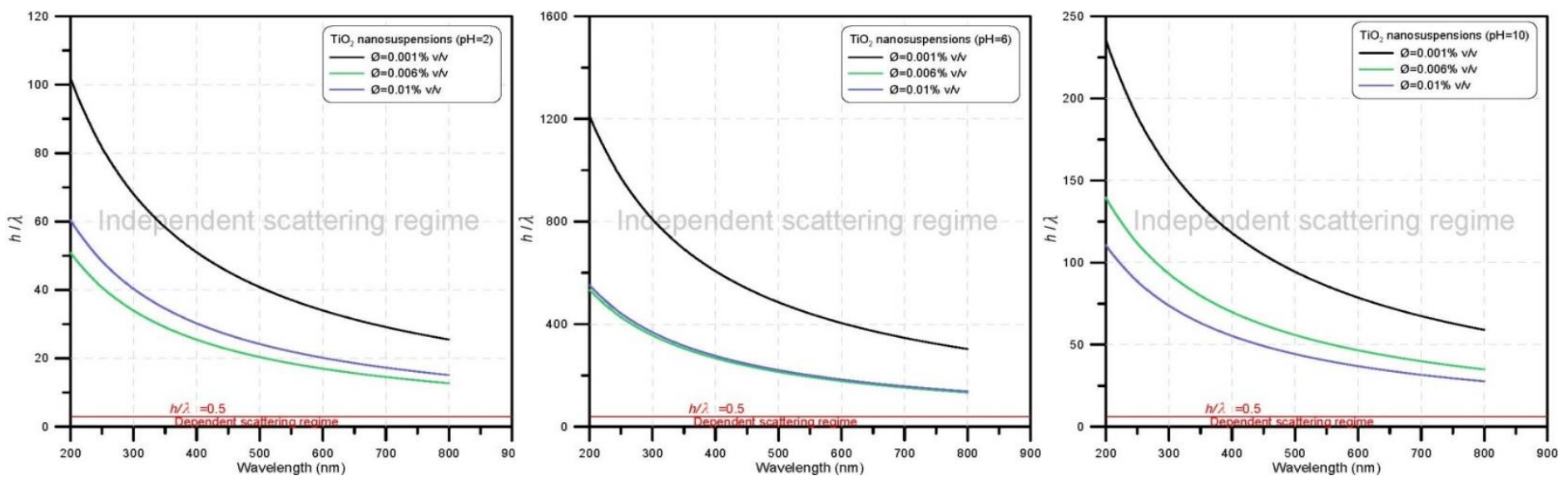

(a) Water based $\mathrm{TiO}_{2}$
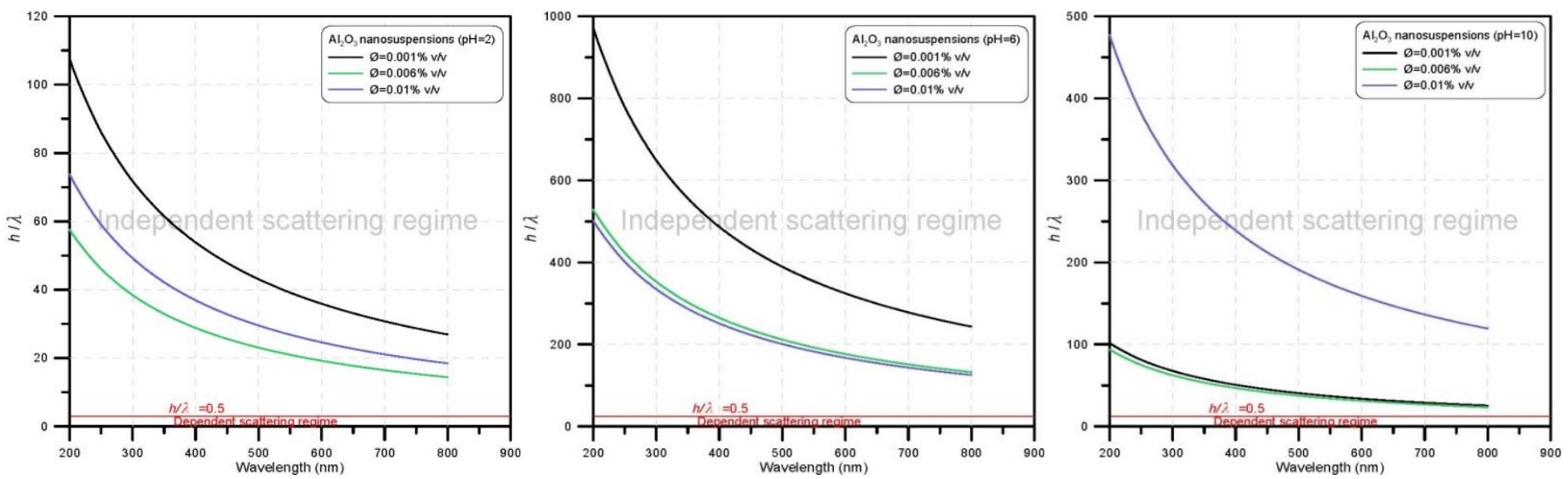

(b) Water based $\mathrm{Al}_{2} \mathrm{O}_{3}$
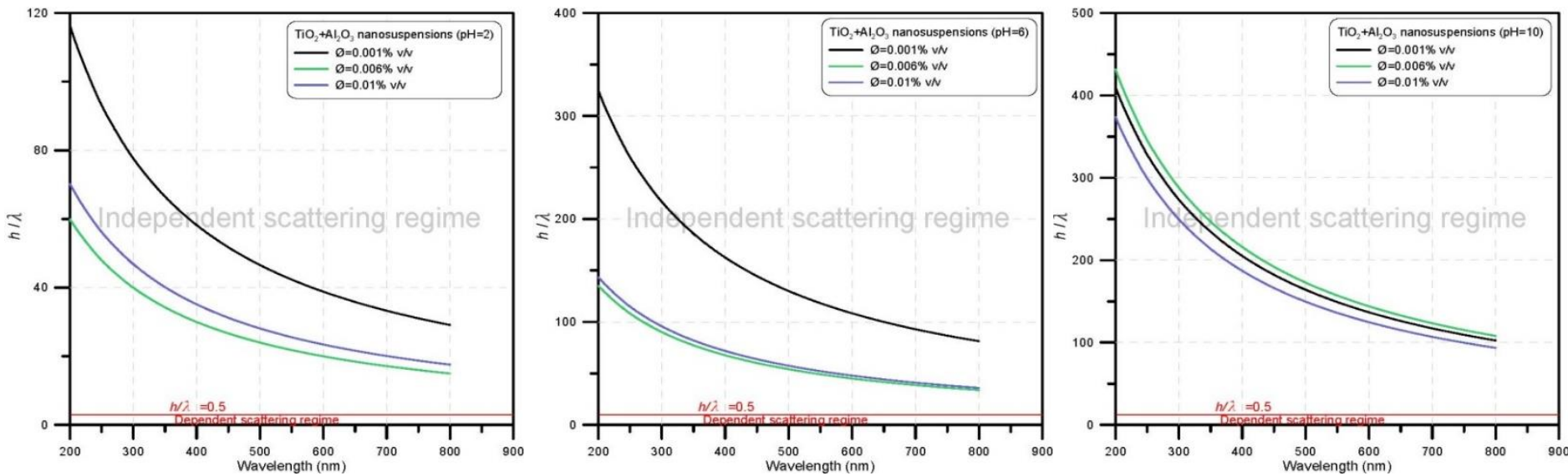

(c) Water based $\mathrm{TiO}_{2}+\mathrm{Al}_{2} \mathrm{O}_{3}$

Figure 8. Boundaries of Independent and dependent scattering regimes curves for nanosuspensions 
Hybrid and $\mathrm{TiO}_{2}$ nanosuspensions exhibit high extinction coefficients in comparison with the other type $\left(\mathrm{Al}_{2} \mathrm{O}_{3}\right.$ nanosuspensions). The effect of the particle volume fraction on the extinction coefficient can be observed, and different extinction coefficients are obtained for the different particle size of the nanosuspensions under the effect of the $\mathrm{pH}$ value.

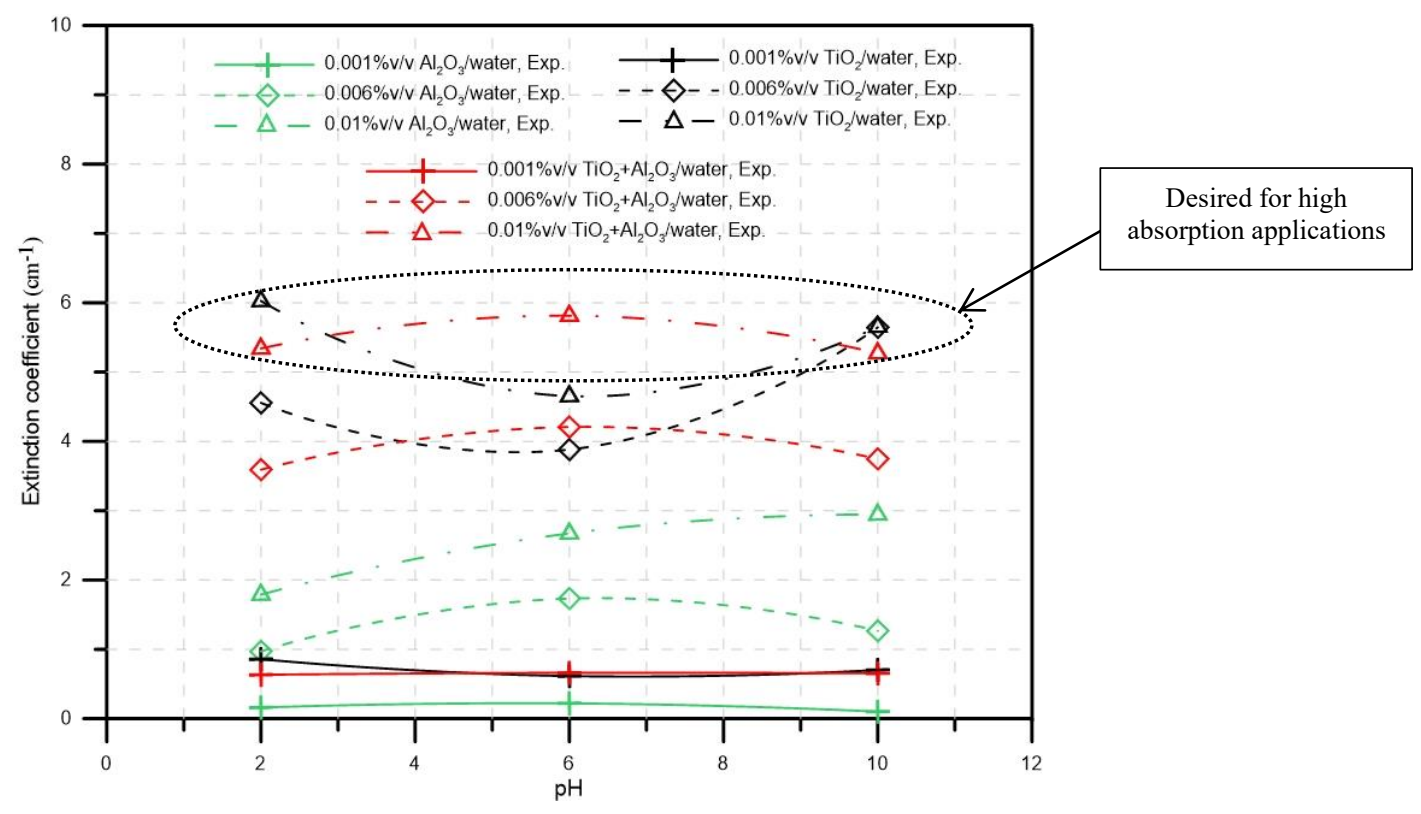

Figure 9. $\mathrm{pH}$ versus extinction coefficient for nanosuspensions at wavelength $=400 \mathrm{~nm}$

The manipulation of the radiative properties of any material no matter whether it is rigid or a particulate medium in the same conditions is very important and is desired in different applications. In this research, different radiative properties are obtained by changing the $\mathrm{pH}$ value of the nanosuspensions in the same conditions (the particle size and shape, and $\emptyset$ ) and in the same medium. In addition, different radiative properties are obtained from the synthesis hybrid system that includes dissimilar particles. As observed from the results in Figs. (5 and 6), the scattering coefficient has a great impact in the extinction coefficient. Therefore, the relationship between the $\mathrm{pH}$ and particle scattering behavior can be observed clearly, which carries significant indications for a specific type of application.

\section{CONCLUSIONS}

Promising applications can be achieved by manipulating the radiative properties of the nanosuspensions with a trade-off between other properties (chemical, and physical, etc.) of the medium and suspended particles, and the goal is to enhance both the radiative properties and particle stability. In this study, the important concepts of particle agglomeration and their effects on radiative properties are explored, which are difficult to detect in theatrical research. The present study shows the effects of dissimilar particles agglomeration (composite agglomerate) on the stability behavior and radiative properties of nanosuspensions, which are important parameters in the radiative transfer phenomena investigations of such type of suspensions. Hybrid nanosuspensions are a new class of suspensions, which can be used to modify the stability and radiative properties of nanosuspensions rather than their individual existence.

This research explains clearly the stability behavior and its effects on the radiative properties of two different types of nanosuspensions (Individual and hybrid). The effect of particle agglomeration on the independent and dependent scattering boundaries is explored. Indeed, the two nanoparticles $\left(\mathrm{TiO}_{2}\right.$ and $\left.\mathrm{Al}_{2} \mathrm{O}_{3}\right)$, which are investigated have their own stability behavior and radiative properties, and they show different stability behavior and radiative properties when they are combined in the same medium (hybrid). $\mathrm{The}^{\mathrm{TiO}_{2}}$ particle shows better radiative 
properties than those of the $\mathrm{Al}_{2} \mathrm{O}_{3}$ particle within the UV/Vis wavelength ranges. On the other hand, these two types of nanoparticles show stability issues at specified $\mathrm{pH}$ values, while the hybrid system $\left(\mathrm{TiO}_{2}\right.$ and $\left.\mathrm{Al}_{2} \mathrm{O}_{3}\right)$ shows a significant enhancement in the stability behavior with good radiative properties at $\mathrm{pH}=2$ and 6 , which is one of the most important observations in this study.

\section{ACKNOWLEDGMENTS}

The authors would like to acknowledge the support that was received from the Center for Energy, Environment, and Economy (CEEE) at Ozyegin University, Istanbul.

\section{REFERENCES}

[1] Souayeh B, Reddy MG, Sreenivasulu P, Poornima T, Rahimi-Gorji M, Alarifi IM. Comparative analysis on non-linear radiative heat transfer on MHD Casson nanofluid past a thin needle. Journal of Molecular Liquids (2019), 284, 163-174. https://doi.org/10.1016/j.molliq.2019.03.151

[2] Raju SS, Kumar KG, Rahimi-Gorji M, Khan I. Correction to: Darcy-Forchheimer flow and heat transfer augmentation of a viscoelastic fluid over an incessant moving needle in the presence of viscous dissipation. Microsystem Technologies. 2019 Mar 26;25(9):3407-.

[3] Duffie JA, Beckman WA. Solar Engineering of Thermal Processes. 2nd edition, John Wiley \& Sons, New York, 1991.

[4] Green MA. Solar Cells: Operation Principles, Technology, and System Applications. Prentice Hall, Englewood Cliffs, NJ, 1981.

[5] Choi C, Yoo HS, Oh JM. Preparation and heat transfer properties of nanoparticle-in-transformer oil dispersions as advanced energy-efficient coolants. Curr Appl Phys 2008;8:710-2. https://doi.org/10.1016/j.cap.2007.04.060

[6] Xuan Y, Li Q. Heat transfer enhancement of nanofluids. Int J Heat Fluid Flow 2000;21:58-64. https://doi.org/10.1016/S0142-727X(99)00067-3

[7] Krishna ChM, ViswanathaReddy G, Souayeh B, Raju CSK, Rahimi-Gorji M, Raju SSK. Thermal convection of MHD Blasius and Sakiadis flow with thermal convective conditions and variable properties. Microsystem Technologies (2019): 1-12. https://doi.org/10.1007/s00542-019-04353-y

[8] Hussanan A, Khan I, Gorji MR, Khan WA. CNTS-Water-Based Nanofluid Over a Stretching Sheet. BioNanoScience, 9(1), 21-29, 2019. https://doi.org/10.1007/s12668-018-0592-6

[9] Choi SUS. Enhancing Thermal Conductivity of Fluids with Nanoparticles, in Developments and Applications of Non-Newtonian Flows. D.A. Siginer and H.P. Wang, eds, FED-Vol. 231/MD-Vol. 66, ASME,1995, U.S., New York, pp 99-105.

[10] Wang BX, Zhou LP, Peng XF. A fractal model for predicting the effective thermal conductivity of liquid with suspension of nanoparticles. International Journal of Heat and Mass Transfer 46, no. 14, 2665-2672, 2003. https://doi.org/10.1016/S0017-9310(03)00016-4

[11] Keblinski P, Cahill DG. Comment on Model for heat conduction in nanofluids. Phys. Rev. Lett. 95, no. 20, 209401, 2005. https://doi.org/10.1103/PhysRevLett.95.209401

[12] Modest MF. Radiative Heat Transfer. Academic Press-Elsevier science, USA, 2003.

[13] Howell JR, Mengüç MP, Siegel R. Thermal Radiation Heat Transfer. 6th edition, CRC Press, New York, 2016.

[14] Russel W B, Saville D A, Schowalter W R. Colloidal Dispersions. Cambridge University Press, NewYork, 1989.

[15] Myers D. Surface, Interfaces and Colloids (Principles and Applications). 2nd edition, John Wiley and Sons., New York, USA, 1999.

[16] Mishchenko MI. Electromagnetic Scattering by Particles and Particle Groups (An Introduction). 1st edition, Cambridge, 2014.

[17] Sarkar J. Acritical review of heat transfer correlations of nanofluids. Renew Sustain Energy Rev 2011; 15:3271-7. https://doi.org/10.1016/j.rser.2011.04.025

[18] Yu W, Xie H. A review on nanofluids: preparation, stability mechanisms, and applications. J Nanomater 2012; 2012: 435873. https://doi.org/10.1155/2012/435873

[19] Wong KV, Leon OD. Applications of nanofluids: current and future. Adv Mech Eng 2010;2010:519659. https://doi.org/10.1155\%2F2010\%2F519659

[20] Das SK, Choi SUS, Patel H E. Heat transfer in nanofluids - a review. Heat Transf Eng 2006;27(10):3-19. https://doi.org/10.1080/01457630600904593 
[21] Wang XQ, Mujumdar AS. Heat transfer characteristics of nanofluids: a review. Int J Therm Sci 2007;46:1-19. https://doi.org/10.1016/j.ijthermalsci.2006.06.010

[22] Daungthongsuk W, Wongwises S. A critical review of convective heat transfer of nanofluids. Renew Sustain Energy Rev 2007;11:797-817. https://doi.org/10.1016/j.rser.2005.06.005

[23] Trisaksria V, Wongwises S. Critical review of heat transfer characteristics of nanofluids. Renew Sustain Energy Rev 2007;11(3):512-23. https://doi.org/10.1016/j.rser.2005.01.010

[24] Otanicar TP, Phelan PE, Prasher RS, Rosengarten G, Taylor RA. Nanofluid based direct absorption solar collector. J. Renew. Sust. Energy 2 (2010) 1-13. https://doi.org/10.1063/1.3429737

[25] Tyagi H, Phelan P, Prasher R. Predicted efficiency of a low-temperature nanofluid-based direct absorption solar collector. J. Sol. Energy Eng. 131 (2009) 0410041-0410047. https://doi.org/10.1115/1.3197562

[26] Luo Z, Wang W, Wei W, Xiao G, Ni M. Performance improvement of a nanofluid solar collector based on direct absorption collection (DAC) concepts. Int. J. Heat Mass Transfer 75 (2014) 262-271. https://doi.org/10.1016/j.ijheatmasstransfer.2014.03.072

[27] Cheng P, Gu MY, Jin YP. Recent progress in titania photocatalyst operating under visible light. Prog. Chem., 17(1), 8-14, 2005.

[28] Ivezic Z, Mengüç MP. An investigation of dependent/independent scattering regimes using a discrete dipole Approximation. Int. J. Heat Mass Transfer. Vol. 39, No. 4, pp. 811-822, 1996. https://doi.org/10.1016/00179310(95)00142-5

[29] Ivezic Z, Mengüç MP, Knauer TG. A procedure to determine the onset of soot agglomeration from multiwavelength experiment. J. Quant. Spectrosc. Radiat. Transfer Vol. 57. No. 6. pp. 859-865. 1997. https://doi.org/10.1016/S0022-4073(97)00001-0

[30] Aslan MM, Mengüç MP, Manickavasagam S, Saltiel C. Size and shape prediction of colloidal metal oxide $\mathrm{MgBaFeO}$ particles from light scattering measurements. Journal of Nanoparticle Research.https://doi.org/ 10.1007/s11051-006-9115-4, 2006.

[31] Viskanta R, Ungan A, Menguc MP. Predictions of radiative properties of pulverized coal and fly-ash polydispersion. Am. Soc. Mech. Eng. ,(Pap.), (United States), Volume: 81-HT-24, 1981.

[32] Kozan M, Thangala J, Bogale R, Mengüç MP, Sunkara M K. In-situ characterization of dispersion stability of WO3 nanoparticles and nanowires. J. Nanopart Res. https://doi.org/ 10.1007/s11051-007-9290-y, Springer, 2007.

[33] Hosokawa M, Nogi K, Naito M, Yokoyama T. Nanoparticle Technology Handbook. Elsevier, 2007.

[34] Shih W, Hirata Y, Carty W. Colloidal Ceramic Processing of Nano-, Micro-, and Macro-Particulate Systems. The American Ceramic Society, 2004.

[35] Tabordaa EA, Francoa CA, Loperab +SH, Alvaradoc V, Cortés FB. Effect of nanoparticles/nanofluids on the rheology of heavy crude oil and its mobility on porous media at reservoir conditions. Fuel, Volume 184, 15 November 2016, Pages 222-232. https://doi.org/10.1016/j.fuel.2016.07.013

[36] Das PK., Mallik AK., Ganguly R, Santra AK. Synthesis and characterization of $\mathrm{TiO}_{2}$-water nanofluids with different surfactants. International Communications in Heat and Mass Transfer, Volume 75, July 2016, Pages 341-348. https://doi.org/10.1016/j.icheatmasstransfer.2016.05.011

[37] Bohren CF, Huffman DR. Absorption and Scattering of Light by Small Particles. Wiley-Interscience Publication, Canada, 1983.

[38] Van de Hulst HC. Light scattering by small particles. Dover publications, Inc., New York, 1981.

[39] Drolen BL, Tien CL. Independent and dependent scattering in packed-sphere systems. J. Thermophysics, Vol. 1, No. 1, January 1987.

[40] Taylor RA, Phelan PE, Otanicar TP, Adrian R, Prasher R. Nanofluid optical property characterization: towards efficient direct absorption solar collector. Nanoscale Research Letters 2011, 6:225. https://doi.org/10.1186/1556-276X-6-225

[41] Woodcock LV. Proceedings of a Workshop held at Zentrum für Interdisziplinäre Forschung. University Bielefield, November 11-13, 1985, Edited by Th. Dorfmüller and G. Williams (Lect. Notes Phys., 277, 113124 (1987)). 